\title{
Pulsar timing with the DFB at Nanshan
}

\author{
J. P. Yuan, N. Wang, Z. Y. Liu, and J. B. Wang \\ Xinjiang Astronomical Observatory, CAS, 150 Science 1-Street, Urumqi, \\ Xinjiang, China, 830011 \\ email: yuanjp@xao.ac.cn
}

\begin{abstract}
Pulsar timing observations are being carried out with the Nanshan 25-metre radio telescope since 2000. We observe about 300 pulsars, including nine millisecond pulsars, at $1.5 \mathrm{GHz}$ with a cryogenic receiver and digital filterbank. Frequent observations at Nanshan revealed 50 glitches. We detect nine more glitches in the past two years. Timing solutions obtained with the Nanshan telescope for eight radio loud Gamma ray pulsars are presented.
\end{abstract}

Keywords. stars: pulsars: general, timing

\section{Introduction}

Pulsar timing has long been used to study the instability of pulsar periods, the distribution and turbulence of the interstellar free electron in Galaxy, as well as pulsar proper motions and hence their velocities. The pulse times of arrival (TOA) of millisecond pulsars (MSP) can be measure much accurately than normal pulsars and their spin is much smoother, make them as better clock. Moreover they are unique objects which can be used to test the relativity theories, detect the gravitational wave background, and study the evolution of binary.

The Nanshan 25-metre radio telescope operated by Xinjiang Astronomical Observatory (XAO), has been dedicated to monitor pulsars since 2000. In 2010 Jan, a Digital FilterBank (DFB) backend became operational; here we show some results of pulsar timing observations performed with it.

\section{Data analysis and Results}

Our observations have been made using a dual-channel cryogenic receiver with a bandwidth of $320 \mathrm{MHz}$ centred at $1540 \mathrm{MHz}$, since 2002 June. There are about 300 pulsars being observed with integration times of $4-16 \mathrm{~min}$ for each pulsar and three sessions per month. The dedispersion is provided by a $1024 \times 0.5 \mathrm{MHz}$ DFB since 2010 . The DFB digitizes band-limited signal in the four Stokes parameters from each of the two orthogonal polarizations. The system sensitivity is $0.4 \mathrm{mJy}$ for $16 \mathrm{~min}$ observing time.

The PSRCHIVE and TEMPO2 packages were used to analyze the data (Hotan et al. 2004, Hobbs et al.2006). Local arrival times were determined by correlating the observed average pulse profiles with standard pulse profiles. The basic timing model for the barycentric pulse phase, $\phi$, as a function of time $t$ is

$$
\phi(t)=\phi_{0}+\nu\left(t-t_{0}\right)+\frac{1}{2} \dot{\nu}\left(t-t_{0}\right)^{2}+\frac{1}{6} \ddot{\nu}\left(t-t_{0}\right)^{3}
$$

where $\phi_{0}$ is the phase at time $t_{0}$, and $\nu, \dot{\nu}, \ddot{\nu}$ represent the pulse frequency, frequency derivative and frequency second derivative.

A comprehensive pulsar monitor program was carried out by the worldwide radio pulsar timing community in support the Fermi Gamma ray pulsar commission (Smith 
Table 1. Timing solutions for eight radio loud Gamma ray pulsars.

\begin{tabular}{lllllll}
\hline PSR J & $\nu$ & $\dot{\nu}$ & $\ddot{\nu}$ & Epoch & Data range & RMS \\
\hline & $(\mathrm{Hz})$ & $\left(\times 10^{-15} \mathrm{~s}^{-2}\right)$ & $\left(\times 10^{-24} \mathrm{~s}^{-3}\right)$ & $(\mathrm{MJD})$ & $(\mathrm{MJD})$ & $(\mu \mathrm{s})$ \\
\hline $0534+2200$ & $29.7021756869(3)$ & $-3706.7919(6)$ & $11470(3)$ & 56015 & $55925-56126$ & 156 \\
$0631+1036$ & $3.4745192097(2)$ & $-1264.293(17)$ & $71(6)$ & 55915 & $55707-56126$ & 1694 \\
$0659+1414$ & $2.59794675570(3)$ & $-370.9272(12)$ & $0.12(17)$ & 55650 & $55202-56126$ & 1196 \\
$0742-2822$ & $5.99622971186(11)$ & $-605.240(4)$ & $3.1(7)$ & 55650 & $55202-56102$ & 1822 \\
$1730-3350$ & $7.1685291201(8)$ & $-4354.75(3)$ & $12(3)$ & 55500 & $55012-56102$ & 8426 \\
$1801-2451$ & $8.002654070(3)$ & $-8184.4(4)$ & $472(20)$ & 55650 & $55666-56127$ & 1620 \\
$1835-1106$ & $6.0270662649(6)$ & $-747.87(3)$ & $-42(4)$ & 55750 & $55307-56129$ & 4730 \\
$2043+2740$ & $10.40245141153(15)$ & $-133.408(13)$ & $18.5(12)$ & 55580 & $55202-56102$ & 1154 \\
\hline
\end{tabular}

et al. 2008). More than 760 pulsar ephemerides from radio observatories are obtained (Abdo et al. 2010), many of these pulsars have high spin-down power $\left(\geqslant 10^{34} \mathrm{erg} / \mathrm{s}\right)$ and suffer from a high degree of timing noise. Xinjiang Astronomical Observatory joined the program, using the 25-metre dish at Nanshan to monitor about 38 pulsars. Table 1 shows the timing solutions of eight radio loud Gamma ray pulsars. All pulsars but PSR J2043+2740 have glitches reported. Besides the instability of pulsar spin, we also measure the flux and polarization of these pulsars, aiming to study the correlation between their Gamma ray and the radio flux, and help us understand the mechanism of pulsar emission.

Frequent observations at Nanshan revealed 50 glitches up to September 2012 (Wang et al. 2001, Zou et al. 2004, 2008, Yuan et al. 2010a, 2010b, Wang et al. 2012). There are more nine glitches that have not been reported in our earlier works (see Table 2 for details). A new glitch was detected in the Crab pulsar (the fractional jump in frequency $\left.\Delta \nu_{\mathrm{g}} / \Delta \nu>34(2) \times 10^{-9}\right)$ in 2011 November with a longer preceding interval of about 1300 days. Figure 1 presents the results of timing analysis of PSR J0631+1036, showing

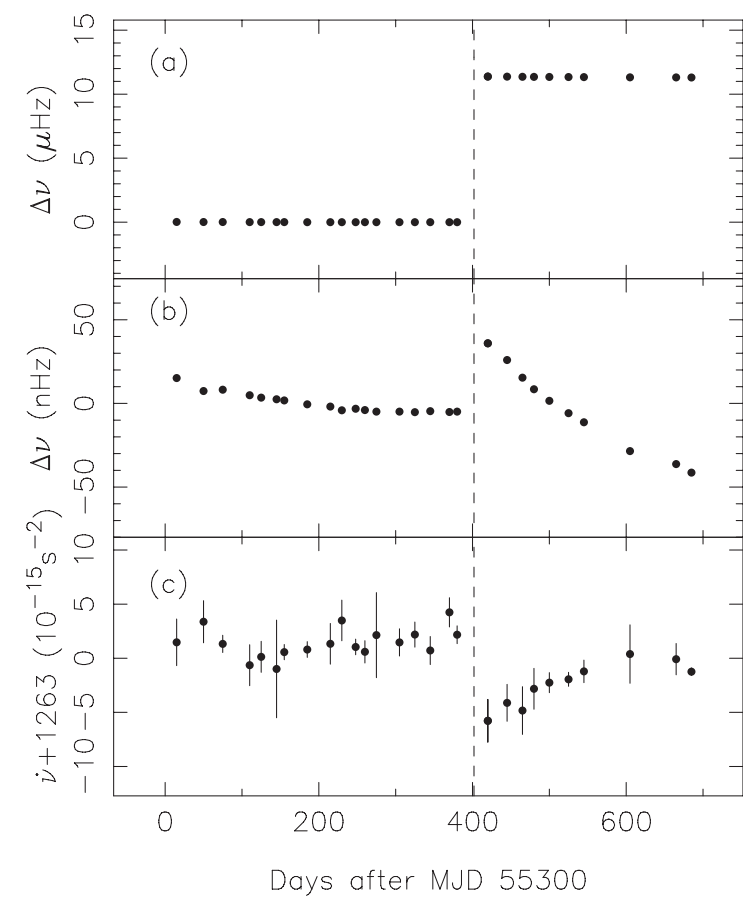

Figure 1. The glitch of PSR J0631+1036. (a) Variations of rotational frequency relative to the pre-glitch solution. (b) An expanded plot of variations of rotational frequency. (c) Variations of rotational frequency derivative. 
Table 2. Parameters of the nine new detected glitches

\begin{tabular}{ccrc}
\hline Psr Name & Glitch Epoch (MJD) & $\Delta \nu_{\mathrm{g}} / \nu\left(\times 10^{-9}\right)$ & $\Delta \dot{\nu}_{\mathrm{g}} / \dot{\nu}\left(\times 10^{-3}\right)$ \\
\hline $\mathrm{J} 0534+2200$ & $55875.5(1)$ & $>34(2)$ & - \\
$\mathrm{J} 0631+1036$ & $55116(4)$ & $7.2(8)$ & $-1.3(9)$ \\
& $55702(3)$ & $3278(2)$ & $1.4(2)$ \\
$\mathrm{J} 0742-2822$ & $55020.0(3)$ & $102.1(1)$ & $4.3(2)$ \\
$\mathrm{J} 0922+0638$ & $55140(9)$ & $1256.7(6)$ & $-0.9(3)$ \\
$\mathrm{J} 1801-2304$ & $53307(16)$ & $493.8(5)$ & $0.4(2)$ \\
$\mathrm{J} 1824-1118$ & $54302(15)$ & $2875.7(8)$ & $-13(4)$ \\
$\mathrm{J} 1952+3252$ & $54104(2)$ & $5.7(7)$ & $-0.5(6)$ \\
$\mathrm{J} 1957+2831$ & $54691(4)$ & $5.7(1)$ & $1.3(3)$ \\
\hline
\end{tabular}

a very large glitch with a frequency jump $\Delta \nu_{\mathrm{g}} \sim 11 \times 10^{-6} \mathrm{~Hz}$ occurred in 2011 . The dashline in Figure 1 indicates the glitch epoch given by the Jodrell Bank glitch catalogue (Espinoza et al. 2011). Most of the jump $\Delta \nu_{\mathrm{g}}$ persist beyond the end of the data span. A phase-coherent fit is consistent with a glitch of $\Delta \nu_{\mathrm{g}} / \Delta \nu \sim 3.278 \times 10^{-6}$ and a decay model with a time constant $\tau_{\mathrm{d}} \sim 160 \mathrm{~d}$. It is generally believed that glitch and post-glitch behavior reflect the dynamics of the interior of the neutron star rather than magnetospheric phenomena. Observing glitches and measuring their subsequent decay processes provide one of the few probes of neutron star structure and thus the physics of ultra-dense matter.

Nine MSPs are observed with the Nanshan radio telescope: PSRs J1022+1001, J1518+ 4904, J1713+0747, J1643-1224, J1600-3053, J1744-1134, J1857+0943, J1939+2134, J2145-0750, seven of which are in binary system except PSRs J1744-1134 and J1939+ 2134. The signal obtained with Nanahsn telescope from PSR J1600-3053 is weak, which has $\mathrm{S} / \mathrm{N}$ of $\sim 10$ with 16 min integration time. The profiles of other eight MSPs have $\mathrm{S} / \mathrm{N}$ of $15-50$. For the TOAs obtained in one year, the minimum value of timing RMS residuals is several microseconds.

\section{Summary}

We have presented the timing solutions of eight radio loud Gamma ray pulsars and nine glitches observed with DFB. As many pulsars have no observed glitch over spans of three decades or more, it is valuable to continue timing observations of a large set of pulsars, in order to better characterize glitch properties and hopefully to lead to a better understanding the mechanisms involved. The large number of timing observation at Nanshan with data spanning $>10 \mathrm{yr}$ also provide tools to study the pulsar timing noise that is present in most pulsars.

\section{References}

Abdo A. A., Ackermann M., Ajello M. et al., 2010 ApJS, 187, 460 Espinoza C. M., Lyne A. G., Stappers B. W., \& Kramer M., 2011, MNRAS, 414, 1679

Hobbs, G. B., Edwards, R. T., \& Manchester, R. N. 2006, MNRAS, 369, 655

Hotan, A. W., van Straten, W., \& Manchester, R. N. 2004, PASA, 21, 302

Smith D. A., Guillemot L., Camilo F. et al., 2008, A\&A A, 492, 923

Wang, N., Manchester, R. N., Zhang, J., et al. 2001, MNRAS, 328, 855

Wang J. B., Wang N., Tong H., \& Yuan J. P., 2012, ApSS, 340, 307

Yuan, J. P., Wang, N., Manchester, R. N., \& Liu, Z. Y. 2010a, MNRAS, 404, 289

Yuan, J. P., Manchester, R. N., Wang, N., et al. 2010b, ApJL, 719, L111

Zou, W. Z., Wang, N., Wang, H. X., et al. 2004, MNRAS, 354, 811

Zou, W. Z., Wang, N., Manchester, R. N., et al. 2008, MNRAS, 384, 1063 